\title{
A European pharmacotherapeutic agent Roflumilast exploring integrated preclinical and clinical evidences for SARS CoV-2 mediated inflammation to organ damage
}

\author{
yogendra Singh ${ }^{1}$, Neeraj Fuloria ${ }^{2}$, Shivkanya Fuloria ${ }^{2}$, Vetriselvan Subramaniyan ${ }^{3}$, Waleed \\ Almalki $^{4}$, Fahad Al-abbasi ${ }^{5}$, Imran Kazmi ${ }^{5}$, Sobhit Rajput ${ }^{6}$, and Gaurav Gupta ${ }^{7}$ \\ ${ }^{1}$ Maharishi Arvind College of Pharmacy \\ ${ }^{2}$ AIMST University \\ ${ }^{3}$ MAHSA University \\ ${ }^{4}$ Umm Al-Qura University \\ ${ }^{5}$ King Abdulaziz University \\ ${ }^{6}$ Aligarh College of Pharmacy \\ ${ }^{7}$ Suresh Gyan Vihar University
}

November 8, 2021

\begin{abstract}
COVID-19 has spread globally, affecting almost 160 million individuals. Elderly and pre-existing patients (such as diabetes, heart disease and asthma), seems more susceptible to serious illness with COVID-19. Roflumilast was licensed for usage in the European Union in July 2010 as a phosphodiesterase-4 (PDE4) inhibitor. Roflumilast has been shown to decrease bleomycininduced lung fibrosis, lung hydroxyproline, right heart thickning in animal prophylactic. The current study reviewed existing data that the PDE-4 inhibitor protects not just renal tissues but also other major organ systems after COVID-19 infection by decreasing immune cell infiltration. These immune-balancing effects of roflumilast were related with a decrease in oxidative and inflammatory burden, caspase-3 suppression, and increased PKA/cAMP levels in renal and other organ tissue.
\end{abstract}

\section{Introduction:}

COVID-19 has spread globally, affecting more than 216,867,420 documented occurrences and 4,507,837 fatalities in individuals. Elderly and pre-existing patients (such as diabetes, heart disease and asthma), seems more susceptible to serious illness with COVID-19. Due to changes in blood glucose and other diabetes-related complications, diabetic individuals who become infected can be hard to treat. A significant comorbidity towards the occurrence of SARS CoV-2-related COVID-19 includes inflammatory cytokine storm, acute kidney injury, myocarditis, thrombosis, ARDS, TIA mediated cerebral complication that ultimately leads to multi-organ failure. A recent case report illustrates an atypical initial presentation and subsequent complications throughout the middle-aged man. The person complained of abdominal discomfort and vomiting then was diagnosed with a severe acute renal damage. During the hospitalization, the study participant sustained a myocardial infarction and respiratory failure. This indicates that this patient most likely developed cardiorenal syndrome as a result of COVID-19-associated acute renal injury. ${ }^{1}$ Notably, the main contributor to the above-mentioned consequences of organ damage and death is inflammation.

In an early stage of SARS CoV-2 attack, pro-inflammatory cytokines circulating including IFN- $\gamma$, IL-1/2/6/8, and $\mathrm{TNF}-\alpha$, directly exacerbate the damage by attracting various leukocyte populations to the site of injury, resulting in a devastating inflammatory pathway. As a result of that excitation of immune cells including the 
natural and acquired host defence systems become overloaded, resulting in the release of more proinflammatory cytokines which are accumulated or cleared from the glomerulus, causing damage to the nephron tubular segments and epithelial cell death mediated metabolic dysregulation. ${ }^{2,}{ }^{3}$ Furthermore, in laboratory experiments, leukocyte-platelet (PLT) function has been proven to be integral to leukocyte recruitment. ${ }^{4}$ and the progression of the vascular inflammation and thrombosis. ${ }^{5}$ In COVID-19 infection, systemic inflammation is linked with a 'procoagulant' condition defined by excessively elevated tissue factor and factor VIIa levels. These findings imply that the prothrombotic activity of circulating leukocytes may contribute to the elevated cardiac, cerebral and renal vascular risk observed in individuals infected with COVID-19. ${ }^{6}$ In the early stage of the SARS CoV-2 assault, hyperinflammatory and activation of the immune system, together with oxidation load lead to various pathological changes in renal tissue, including cell proliferation, podocyte (proteinuria), deposition of ECM (extracellular matrix), pro apoptotic factor activation. While the immunosuppression found in the late phase of COVID-19 disease/drug (steroids) associated impairs the body's defenses, aggravating organ damage. ${ }^{7}$ In addition, the peripheral blood count in SARS CoV-2 patients for monocytes and neutrophils in this comparable time frame suggests immune system dysregulation at the late COVID 19 stage. In addition, the peripheral blood count in SARS CoV-2 patients for monocytes and neutrophils in this comparable time frame suggests immune system dysregulation at the late COVID 19 stage. $^{8}$ Additionally, we observed an elevated concentration of plasma leucocytes (CD10 and CD16, for instance) and abnormal blood neutrophil motility, both of which indicate an increased risk of mortality. ${ }^{8,9}$

Roflumilast was licensed for usage in the European Union in July 2010 as a phosphodiesterase-4 (PDE4) inhibitor. Roflumilast has been approved as a 'constraint' treatment to bronchodilators by the EMA summary and, under the GOLD guidelines, an effect also exists in patients not 'controlled on the combination of Fixed Dose LABAs like formoterol and ICS such as beclomethasone dipropionate. Moreover, roflumilast coadministration with budesonide in Asian and Caucasians did not affect the state of steady disposition of either drugs and had no consequences for its safety or tolerability which also denote that it may be consumed without or with meal. ${ }^{10}$ However, roflumilast cessation might be because of the medicine that is relatively costly and, subsequent to complex prescription procedures or unpleasant effects, poor understanding of roflumilast indications between the clinicians. ${ }^{11}$ Recently a study results show that roflumilast protects from cerebral ischemia functional sequelae, which may be connected to its anti-inflammatory characteristics. ${ }^{12}$ Another research also shows in particular that roflumilast inhibitor of PDE-4 prevents LPS from releasing NO, IL-1 $\beta$, TNF- $\alpha$ from the production of the macrophage through suppression of activation of SAPK/JNK, p38 MAPK, NF-kB mechanisms. ${ }^{13}$ Roflumilast has been shown to decrease bleomycin-induced lung fibrosis, lung hydroxyproline, right heart thickning in animal prophylactic and curative trials; it has also been shown to prevent intracinary pulmonary artery muscularization. The inhibitor PDE4 was utilised in the bronchoalveolar lavage fluid to diminish bleomycin-induced transcripts for Tumor necrosis factor - alpha, transforming growth factor-beta, connective tissue growth factor synthesis, endothelin-1, aI(I)collagen, and mucin Muc5ac in the lung, as well as to decrease the levels of IL-13, Tumor necrosis factor - alpha, and Transforming growth factor-beta. Moreover, lung fibrosis, al(I)collagen, right heart thickness generated by bleomycin have been reduced with roflumilast, but not dexamethasone group. ${ }^{14}$ Additionally, another study found that roflumilast and roflumilast $\mathrm{N}$-oxide suppressed macrophages' release like cell attracting molecules and TNF-alpha release in lungs. ${ }^{15}$

Inflammation and immunological response are strongly related to increased PDE-4 synthesis. There are five major PDE-4 isoforms. They are PDED-4A, PDED-4B, PDED-4C, PDED-4D, and PDED-4E. PDE-4B is highly upregulated on neutrophils and monocytes, resulting in the discharge of many inflammation-inducing agents. ${ }^{16}$ In contrast to earlier experimental findings, where LPS injection was shown to stimulate TNF production from circulating leukocytes in PDE-4B knockout mice, it appears that this transcript detrimental effect in inflammatory conditions has been completely inhibited. ${ }^{17}$ Additionally, suppression of PDE-4B repressed apoptotic cell death and inflammation molecule biosynthesis in renal tubular epithelial cell when treated with cisplatin. Previously, when ovalbumin was given to rats' bronchiolar lavage fluid, PDE-4B/D expression was found to be greater than in that of controls. The higher expression of these isoforms was noted in only the lavage fluid of rats with ovalbumin exposure, however, the overexpression of PDE- $4 \mathrm{~B}$ only 
helped rescue allergic symptoms in this study. ${ }^{18}$ The high-dose Roflumilast significantly reduced PDE-4B/D transcripts, suggesting key involvement of these two isoforms in ARDS linked with COVID-19. These data further indicate that inhibitions of both transcripts are adequate to raise cAMP levels in renal tissue that have a protective impact on the kidney. Pre-treatment dosage of Roflumilast addictively decreased renal oxidative stress, inflammatory cytokines, and renal tissue MPO expression. The MPO neutrophil enzyme is strongly pro-oxidizing and pro-inflammatory. ${ }^{19}$ Thus, the inhibitory effects of roflumilast on inflammatory and oxidative injury in COVID-19 infection-induced acute renal injury may be due to the fact that neutrophil recruitment and migratory restriction. Additionally, a research indicates that roflumilast may be helpful in atherothrombotic disorders as well as inflammatory vasculitis that is not primarily associated with lung damage. ${ }^{20}$ Notably, Roflumilast was found to improve glucose uptake and insulin sensitivity. This was linked with stimulation of PKA/cAMP/CREB axis, that leads to PCG-1-dependent activation of mitochondrial energy production.Fig. 1 Depicts the Possible Role of Roflumilast in SARS CoV-2 Mediated Inflammation to Organ Damage

The current study reviewed existing data that the PDE-4 inhibitor protects not just renal tissues but also other major organ systems after COVID-19 infection by decreasing immune cell infiltration. These immunebalancing effects of roflumilast were related with a decrease in oxidative and inflammatory burden, caspase-3 suppression, and increased PKA/cAMP levels in renal and other organ tissue. The findings observations provide fresh light on the late phase events associated with COVID-19-related inflammation and the mechanisms behind roflumilast's organ protective effects.

Acknowledgment: Not Applicable.

Funding: There was no specific funding for this study.

Competing Interests: The authors declare no competing interest.

Availability of data and material : No datasets were generated or analyzed during the current study.

\section{Author Contributions}

Y.S., N.K.F. and S.F. proposed a study; V.S. and W.H.A. participated in all important intellectual content section of the study; F.A.A. and I.K. prepared figures; S.S.R. and G.G. proofread the whole manuscript. All authors approved the content of the manuscript.

\section{Ethics Declarations}

Ethical Approval: Not Applicable

Consent to Participate: Not Applicable

Consent to Publish: Not Applicable

\section{References:}

1. Daniels NF, Ridwan R. Acute kidney injury precipitating myocardial injury in a young male with COVID19. Intern Med J. 2021;51(5):816-817. https://doi.org/https://doi.org/10.1111/imj.14977

2. Zarbock A, Gomez H, Kellum JA. Sepsis-induced acute kidney injury revisited: pathophysiology, prevention and future therapies. Curr Opin Crit Care. 2014;20(6):588-595. https://doi.org/10.1097/MCC.0000000000000153

3. de Pablo R, Monserrat J, Prieto A, Alvarez-Mon M. Role of circulating lymphocytes in patients with sepsis. Biomed Res Int. 2014;2014(671087. https://doi.org/10.1155/2014/671087

4. Smyth SS, Reis ED, Zhang W, et al. Beta(3)-integrin-deficient mice but not P-selectin-deficient mice develop intimal hyperplasia after vascular injury: correlation with leukocyte recruitment to adherent platelets 1 hour after injury. Circulation. 2001;103(20):2501-2507. https://doi.org/10.1161/01.cir.103.20.2501 
5. von Brühl ML, Stark K, Steinhart A, et al. Monocytes, neutrophils, and platelets cooperate to initiate and propagate venous thrombosis in mice in vivo. J Exp Med. 2012;209(4):819-835. https://doi.org/10.1084/jem.20112322

6. McFadyen JD, Stevens H, Peter K. The Emerging Threat of (Micro)Thrombosis in COVID-19 and Its Therapeutic Implications. Circ Res. 2020;127(4):571-587. https://doi.org/doi:10.1161/CIRCRESAHA.120.317447

7. Tay MZ, Poh CM, Rénia L, MacAry PA, Ng LFP. The trinity of COVID-19: immunity, inflammation and intervention. Nat Rev Immunol. 2020;20(6):363-374. https://doi.org/10.1038/s41577-020-0311-8

8. Reusch N, De Domenico E, Bonaguro L, et al. Neutrophils in COVID-19. Front Immunol. 2021;12(952). https://doi.org/10.3389/fimmu.2021.652470

9. Liao M, Liu Y, Yuan J, et al. Single-cell landscape of bronchoalveolar immune cells in patients with COVID-19. Nat Med. 2020;26(6):842-844. https://doi.org/10.1038/s41591-020-0901-9

10. Hermann R, Siegmund W, Giessmann T, et al. The oral, once-daily phosphodiesterase 4 inhibitor roflumilast lacks relevant pharmacokinetic interactions with inhaled budesonide. J Clin Pharmacol. 2007;47(8):10051013. https://doi.org/10.1177/0091270007300950

11. Salvesen ØNU, Davidsen JR, Pottegård A, Henriksen DP. Roflumilast Usage from 2010 to 2016: A Danish Nationwide Drug Utilization Study. Basic Clin Pharmacol Toxicol. 2018;123(3):314-319. https://doi.org/https://doi.org/10.1111/bcpt.13014

12. Bonato JM, Meyer E, de Mendonça PSB, et al. Roflumilast protects against spatial memory impairments and exerts anti-inflammatory effects after transient global cerebral ischemia. Eur J Neurosci. 2021;53(4):11711188. https://doi.org/10.1111/ejn.15089

13. Kwak HJ, Song JS, Heo JY, et al. Roflumilast inhibits lipopolysaccharide-induced inflammatory mediators via suppression of nuclear factor-kappaB, p38 mitogen-activated protein kinase, and c-Jun NH2-terminal kinase activation. J Pharmacol Exp Ther. 2005;315(3):1188-1195. https://doi.org/10.1124/jpet.105.092056

14. Cortijo J, Iranzo A, Milara X, et al. Roflumilast, a phosphodiesterase 4 inhibitor, alleviates bleomycininduced lung injury. Br J Pharmacol. 2009;156(3):534-544. https://doi.org/10.1111/j.1476-5381.2008.00041.x

15. Buenestado A, Grassin-Delyle S, Guitard F, et al. Roflumilast inhibits the release of chemokines and TNF$\alpha$ from human lung macrophages stimulated with lipopolysaccharide. Br J Pharmacol. 2012;165(6):18771890. https://doi.org/10.1111/j.1476-5381.2011.01667.x

16. Wang P, Wu P, Ohleth KM, Egan RW, Billah MM. Phosphodiesterase 4B2 is the predominant phosphodiesterase species and undergoes differential regulation of gene expression in human monocytes and neutrophils. Mol Pharmacol. 1999;56(1):170-174. https://doi.org/10.1124/mol.56.1.170

17. Jin SL, Conti M. Induction of the cyclic nucleotide phosphodiesterase PDE4B is essential for LPS-activated TNF-alpha responses. Proc Natl Acad Sci U S A. 2002;99(11):7628-7633. https://doi.org/10.1073/pnas.122041599

18. Zheng XY, Chen JC, Xie QM, Chen JQ, Tang HF. Anti-inflammatory effect of ciclamilast in an allergic model involving the expression of PDE4B. Mol Med Rep. 2019;19(3):1728-1738. https://doi.org/10.3892/mmr.2019.9802

19. Souto FO, Alves-Filho JC, Turato WM, et al. Essential role of CCR2 in neutrophil tissue infiltration and multiple organ dysfunction in sepsis. Am J Respir Crit Care Med. 2011;183(2):234-242. https://doi.org/10.1164/rccm.201003-0416OC

20. Totani L, Amore C, Di Santo A, et al. Roflumilast inhibits leukocyte-platelet interactions and prevents the prothrombotic functions of polymorphonuclear leukocytes and monocytes. J Thromb Haemost. 2016;14(1):191-204. https://doi.org/https://doi.org/10.1111/jth.13173 


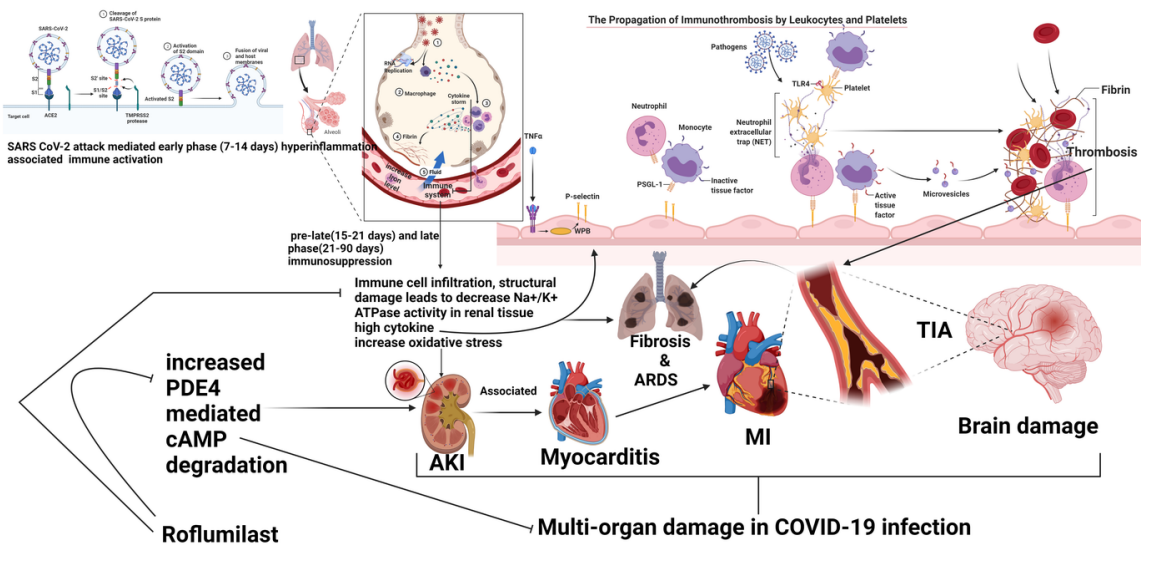

